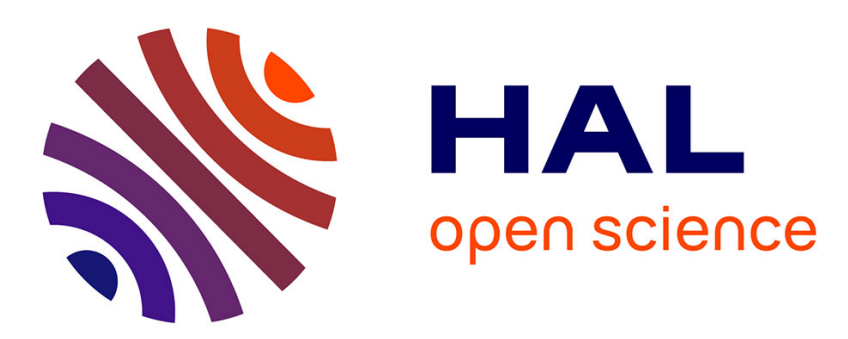

\title{
Learning a reconnecting regularization term for blood vessel variational segmentation
}

Sophie Carneiro Esteves, Antoine Vacavant, Odyssée Merveille

\section{To cite this version:}

Sophie Carneiro Esteves, Antoine Vacavant, Odyssée Merveille. Learning a reconnecting regularization term for blood vessel variational segmentation. IEEE-EMBS International Conference on Biomedical and Health Informatics (BHI), Jul 2021, virtuel, France. hal-03278387

\section{HAL Id: hal-03278387 \\ https://hal.science/hal-03278387}

Submitted on 5 Jul 2021

HAL is a multi-disciplinary open access archive for the deposit and dissemination of scientific research documents, whether they are published or not. The documents may come from teaching and research institutions in France or abroad, or from public or private research centers.
L'archive ouverte pluridisciplinaire HAL, est destinée au dépôt et à la diffusion de documents scientifiques de niveau recherche, publiés ou non, émanant des établissements d'enseignement et de recherche français ou étrangers, des laboratoires publics ou privés. 


\title{
Learning a reconnecting regularization term for blood vessel variational segmentation
}

\author{
Sophie Carneiro Esteves ${ }^{\star}$, Antoine Vacavant ${ }^{\star}$, Odyssée Merveille ${ }^{\dagger}$ \\ * Université Clermont Auvergne, CNRS, SIGMA Clermont, Institut Pascal F-63000, Clermont-Ferrand, France \\ $\dagger$ Univ Lyon, INSA-Lyon, Université Claude Bernard Lyon 1, UJM-Saint Etienne, CNRS, Inserm, CREATIS UMR 5220, U1206, F-69100, \\ LYON, France \\ sophie.carneiro@creatis.insa-lyon.fr,antoine.vacavant@uca.fr,odyssee.merveille@creatis.insa-lyon.fr
}

\begin{abstract}
The segmentation of blood vessels in medical images is a challenging task as they are thin, connected and tortuous. The detection of a connected vascular network is of the utmost importance in clinical applications (e.g. blood flow simulations, vascular network modeling and analysis). Deep learning approaches have been developed to tackle this issue, but they require a large annotated dataset for each new application of interest, which is very challenging to build for vascular networks. In this work, rather than learning the segmentation task, we propose to learn a reconnecting regularization term that learns geometric properties of vascular networks independent of the image modality. Therefore, this term generalizes better than deep learning segmentation models, and can be easily plugged into variational segmentation frameworks to detect vascular networks in different datasets without requiring annotations. We apply this approach on retinal images by training our reconnecting term on the STARE dataset and applying it on the DRIVE dataset. We show that our approach better preserves the connectivity of vascular networks than classic regularization terms in the literature. Finally, we illustrate the generalization power of our reconnecting term by applying it to other types of data.

Index Terms-blood vessel segmentation, reconnection, deep learning , variational approach
\end{abstract}

\section{INTRODUCTION}

The detection of blood vessels in medical images is the key task of most vascular network analysis tools that help clinicians diagnose and treat vascular diseases. Despite being studied for the past twenty years [1], the segmentation of blood vessels is still an open problem as vascular networks are thin, connected, tortuous, sparse and low-contrasted structures.

In this work, we address the important problem of blood vessel connectivity loss upon segmentation of vascular networks. Preserving connectivity is key, as many subsequent processing tasks rely on a connected network such as blood flow simulation or vascular network modeling. Previous work have focused on this issue. Tracking strategies (see [1] Section 7) intrinsically preserve the connectivity of blood vessels by recruiting neighbors pixels recursively, but usually require to be initialized with manual information. Other methods estimate the local direction of blood vessels in order to guide the segmentation in the vessel direction [2, 3].

This work was supported by the LABEX PRIMES (ANR-11-LABX-0063) of Universite de Lyon, and by the IDEXLYON project within the program "Investissements d'Avenir" (ANR-11-IDEX-0007 and ANR-16-IDEX-0005) operated by the French National Research Agency (ANR).
Recently, deep learning strategies have been proposed to segment vascular networks. Some of them aim at enforcing the network connectivity by using topological loss [4] or by computing both the segmentation and centerline of the vessels with the same network [5]. These strategies yield interesting results, but require a large annotated dataset to provide good results. By contrast, variational segmentation strategies do not require annotated data, but are less flexible to represent complex information such as connectivity. To combine the strengths of both methods, decoupled approaches [6] were proposed. They consist in plugging an external operator to act as a regularization term in a variational framework. Early works used denoising algorithms such as BM3D or Non Local Means [7] to replace classic denoising regularization terms such as the Total Variation (TV). More recent strategies learn the external regularization term [8,9] to take advantage of the power representation of deep learning.

In this work, we propose to learn a regularization term designed to preserve the connectivity of vascular networks, and plug it into a variational iterative segmentation framework which does not require annotated data. We show that this regularization term better preserves the connectivity of blood vessels than classic regularization terms such as TV, and more complex ones including directional information.

\section{MATERIALS AND METHODS}

\section{A. Learning a reconnecting term}

We propose to learn a reconnecting network by training a model to reconnect blood vessels from images with artificial disconnections. This network takes as input a binary image of disconnected blood vessels and outputs an image with reconnected vessels. We chose to work on binary images for two reasons. First, creating realistic disconnections is easier on binary images. Second, our goal is to learn a reconnecting term independent of the dataset to apply it on new data for which ground-truths are not available. Learning on binary images is then a better strategy, as the model cannot learn any intensitybased feature of the training dataset.

We first created an algorithm to simulate realistic disconnections. It creates random disconnections in blood vessels with a thickness-dependent probability (the thinner the vessel, the higher the disconnection probability). The disconnections have a random shape to emulate disconnection due to image 

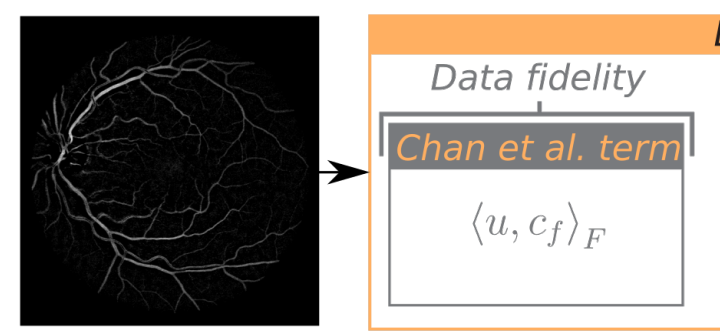

Decoupled approach
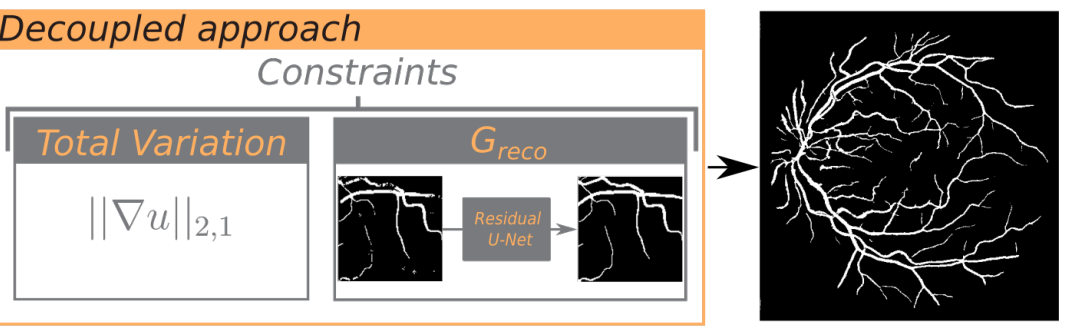

Fig. 1. Pipeline of our proposed method. A reconnecting network is first trained on a separate dataset based on a residual U-Net architecture. Then, a variational segmentation approach is used with the Chan [10] data fidelity term and either the total variation regularization term only or both the total variation and our proposed reconnecting term.

noise. Finally, some artefacts have been added to make the model distinguish parts of vessels that should be reconnected and noise that should not be connected to the vascular network. An example of disconnected image generated by this algorithm is presented in Figure 1

To learn the reconnecting term, the residual U-Net [11] from Monai 1 , a Pytorch-based framework has been used. UNet shows great performance in medical applications and the residual units increase its robustness to image variations. 20 segmentation ground-truths from the STARE [12] dataset were used to create pairs of connected/disconnected vascular images to learn our reconnecting regularization term. We performed data augmentation by rotating each image 4 times and applying on each one different disconnections with our algorithm. This resulted in a dataset of 80 images. 2 images were kept for validation and the rest was used for training. We chose to input patches of size $96 \times 96$ instead of full images to the model to avoid learning the global architecture of the vascular network instead of the reconnecting feature. Additional random rotations and flips were applied on each patch and at each epoch to increase variability and prevent overfitting. A maximum of 1000 epochs, a batch size of 64 , an Adam optimizer with a learning rate of about $10^{-3}$ and a Dice loss were chosen.

\section{B. Variational approach}

Segmentation may be formulated as a variational problem consisting in the optimization of a two-term energy:

$$
\hat{u}=\underset{u \in[0,1]^{N}}{\operatorname{argmin}} E_{\text {data }}(u, f)+\lambda E_{\text {reg }}(u),
$$

where $f \in \mathbb{R}^{N}$ is the initial image, $E_{\text {data }}$ is the data fidelity, $E_{r e g}$ the regularisation term and $\lambda \in \mathbb{R}$ a regularization coefficient acting as a trade-off between both terms. The form of $E_{\text {data }}$ depends on the choice of application. For segmentation the Chan et al. term [10] is classically used:

$$
\begin{aligned}
& E_{\text {data }}(u, f)=\left\langle u, c_{f}\right\rangle_{F}, \\
& c_{f} \mapsto\left(c_{1}-f\right)^{2}-\left(c_{2}-f\right)^{2},
\end{aligned}
$$

where $c_{1}$ and $c_{2}$ are constant values corresponding to the mean background and foreground intensity of the initial image, $\langle., .\rangle_{F}$ is the Frobenius product.

\footnotetext{
${ }^{1}$ https://monai.io/start.html
}

$E_{\text {reg }}$ constrains the problem with regularization and/or prior knowledge. A well-known term is the Total Variation (TV), which reduces the noise in the image, and is defined as follows:

$$
T V(u)=\lambda\|\nabla u\|_{2,1},
$$

with $\|.\|_{2,1}$ the $l_{1}$ norm of $l_{2}$ norm. To solve Eq (1) with a forward-backward primal-dual algorithm (FBPD) [13], we can reformulate the model as follows:

$$
\hat{u}=\underset{u}{\operatorname{argmin}} h(u, f)+g(L u)+k(u),
$$

$h(u, f)=\left\langle u, c_{f}\right\rangle_{F}, g()=.\lambda\|\cdot\|_{2,1}, L=\nabla$ and $k(u)=$ $\iota_{u \in[0,1]^{N}}$ is the indicator function of the set $[0,1]^{N}$. The FBPD algorithm is then :

$$
\begin{aligned}
& u_{i+1}=\operatorname{prox}_{\tau k}\left(u_{i}-\tau\left(\nabla h\left(x_{i}\right)+L^{T} v_{i}\right)\right) \\
& v_{i+1}=\operatorname{prox}_{\sigma g^{\star}}\left(v_{i}+\sigma L\left(2 u_{i+1}-u_{i}\right)\right),
\end{aligned}
$$

where $\operatorname{prox}_{g}$ is the proximity operator of $g, g^{\star}$ is the conjugate of $g, \tau \in \mathbb{R}^{+}$and $\sigma \in \mathbb{R}^{+}$are step-size parameters.

\section{Decoupled approach}

Our reconnecting term takes binary input images and returns a more connected vascular network. We thus propose to plug this reconnecting term after a few iterations of the algorithm presented in Equation 5, when the result $u$ is close to a binary image. We thus replace, in $\mathrm{Eq} 5 \operatorname{prox}_{\sigma k}($.$) by \Phi($.$) defined as$ follows:

$$
\Phi(u)=\left\{\begin{array}{l}
\operatorname{prox}_{\sigma k}(u) \text { if } i<\alpha \\
\mathrm{G}_{\mathrm{reco}}(u) \text { otherwise }
\end{array}\right.
$$

where $G_{\text {reco }}$ is the learnt reconnecting term, $\alpha \in \mathbb{N}^{+*}$ is the iteration number from which the reconnecting term is applied. Algorithm 1 present our proposed algorithm.

\section{EXPERIMENTS}

We applied our approach on the test set of the DRIVE dataset [14], another retinal image dataset. We compared the results with the classic Chan et al. model [10], and the more recent directional total variation model [2]. We optimized the parameters of both compared methods on the DRIVE training set and selected the parameters yielding the best mean results. We set the parameters of our approach experimentally to $\alpha=350$ and $\lambda=0.005$. 


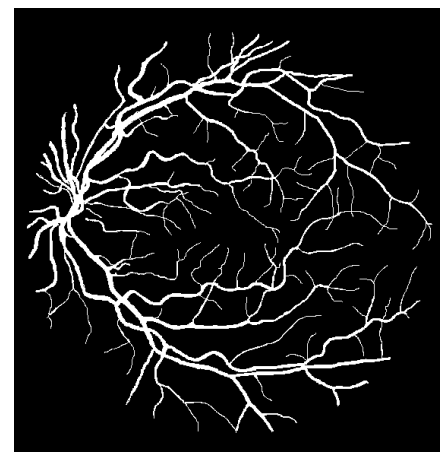

(a) Ground-truth

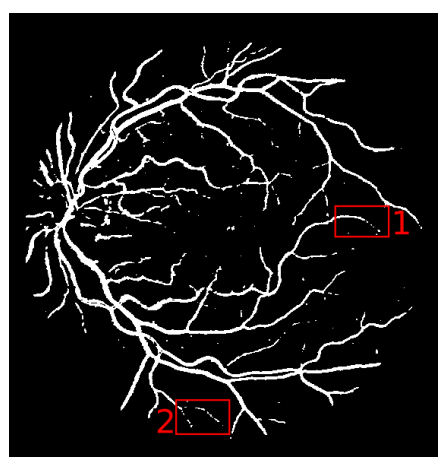

(b) Chan model

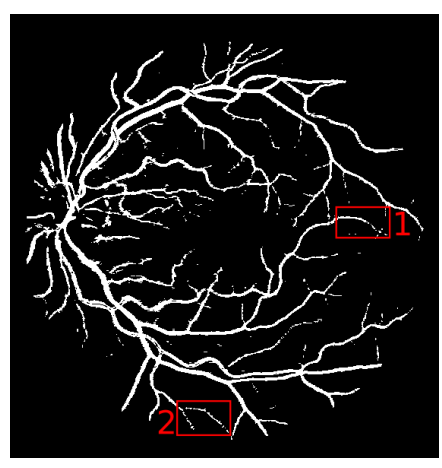

(c) Directional TV

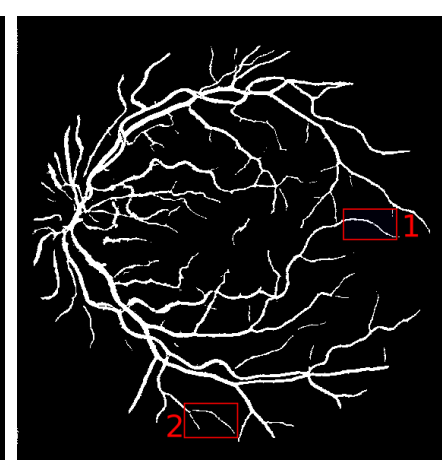

(d) Proposed method

Fig. 2. Comparison of segmentation results on one DRIVE image. Zooms in the red boxes are presented in Figure 3
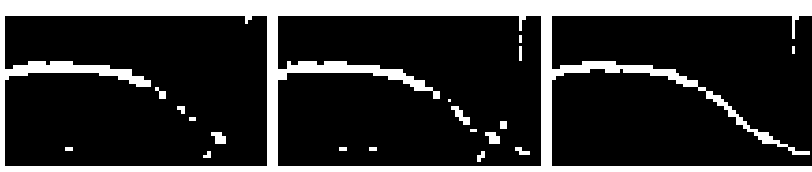

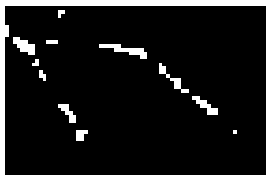

(d) Chan model

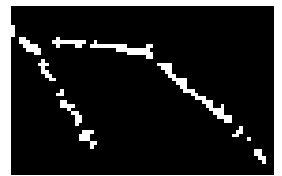

(e) Directional TV

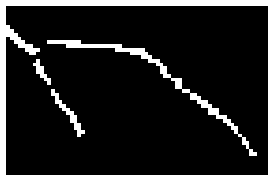

(f) Proposed method
Fig. 3. Zoom of results from Figure 2 Top row corresponds to box 1 and bottom row to box 2 .

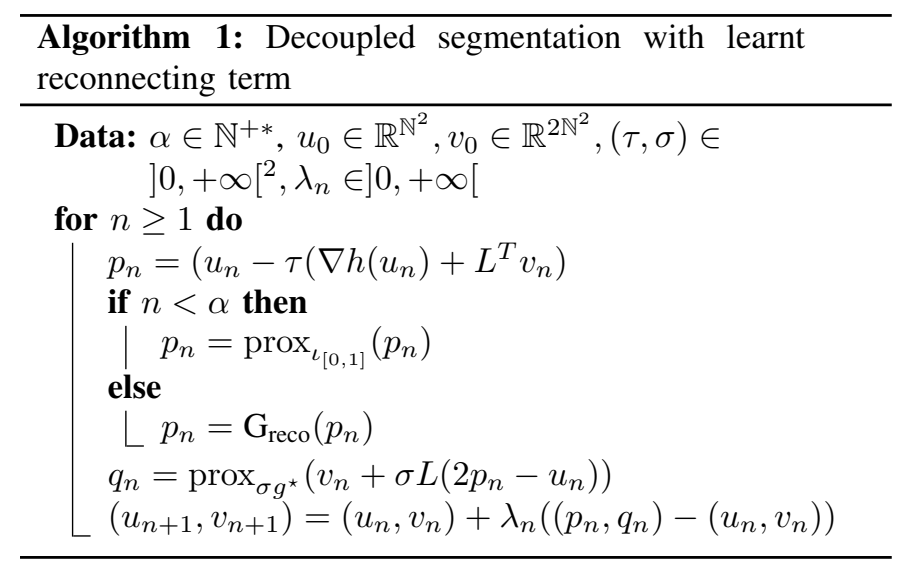

We performed a quantitative evaluation of the results by computing the mean sensitivity (TPR), specificity (TNR) and accuracy (Acc) over the 20 images of the test set (see Eq. 77). All metrics were computed in the field of view provided in the dataset for a fair comparison with the literature results.

$$
\begin{aligned}
& \mathrm{TPR}=\frac{\mathrm{TP}}{\mathrm{TP}+\mathrm{FN}} ; \quad \mathrm{TNR}=\frac{\mathrm{TN}}{\mathrm{TN}+\mathrm{FP}}, \\
& \mathrm{Acc}=\frac{\mathrm{TN}+\mathrm{TP}}{\mathrm{TP}+\mathrm{TN}+\mathrm{FN}+\mathrm{FP}},
\end{aligned}
$$

with TP (resp. TN) the number of true positives (resp. negatives), and FP (resp. FN) the number of false positives (resp. negatives).
The quantitative results are shown in Table IIII We also included the quantitative results from a recent deep learning approach [15] as a comparison. Illustrations of the results are presented in Fig. 2 and Fig. 3

Qualitatively, we observe that our reconnecting term increases the connectivity of the whole vascular network. It also yields a better connectivity than the directional total variation. Moreover, blood vessels seem smoother than the ones obtained with the other two methods, which is for instance an appealing feature for modeling and blood flow simulations. Our results also exhibit less noise, as evidenced by the higher TNR. However, the mean TPR of our approach is lower than for directional TV. We observed that directional TV tends to detect fragmented vessel parts at the extremities of the network. Such fragments are irrelevant for applications requiring connectivity. By contrast, our approach tends to only preserve connected vessels: we found that our approach detected $230 \%$ less connected components than the directional TV approach. This translates into a lower number of true positives, but a better overall quantitative performance and qualitative results for connectivity-requiring applications.

We added the quantitative results from a recent deep learning approach [15] as a baseline for comparison. These approaches are trained with images from the same dataset and thus yield better results. However, these methods do not generalize well on new data (e.g. blood vessels from another organ or another modality). They need to be retrained on each new dataset with their corresponding annotations. Conversely, our approach only requires annotations to train the reconnecting term. Once it is trained, it can be applied in the variational segmentation framework on new data without requiring annotations. To show the power of generalization of our method, we applied the same reconnecting term used on the DRIVE dataset to a different type of image containing blood vessel-like structures. Figure 4 shows the segmentation results on this image. We observe that even though the initial image is different from the STARE dataset, our reconnecting term generalizes well and is able to reconnect the structures of interest whereas a trained U-Net has more difficulties to generalize to this new image type. In this case it seems that 


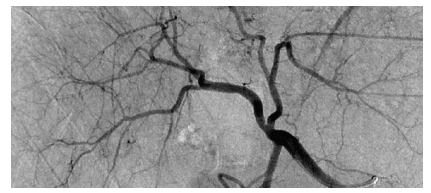

(a) Initial image

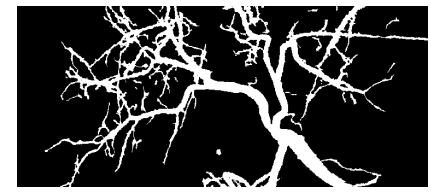

(b) Proposed method

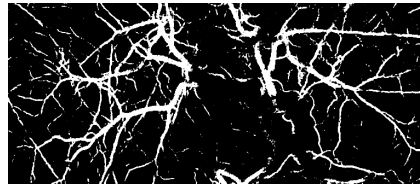

(c) U-Net
Fig. 4. Illustration of the generalization power of our reconnecting term.

TABLE I

QUANTITATIVE SEGMENTATION RESULTS ON THE DRIVE DATABASE.

\begin{tabular}{c|c|c|c} 
& TPR & TNR & Acc \\
\hline \hline Chan model \10 & 0.6615 & 0.9833 & 0.9423 \\
Directional TV model $[2\rceil$ & $\mathbf{0 . 6 8 2 2}$ & 0.9809 & 0.9427 \\
Our approach & 0.6631 & $\mathbf{0 . 9 8 3 9}$ & $\mathbf{0 . 9 4 2 9}$ \\
\hline Deep learning approach (Arias et al. $\llbracket 15])$ & 0.8597 & 0.9690 & 0.9563
\end{tabular}

the network is not able to generalize on images with a larger range of vessel diameters.

\section{CONCLUSION}

We developed a reconnecting term for blood vessel segmentation. This term learns to reconnect blood vessels by focusing on their geometry instead of learning grey-level features in the image. By plugging this term into a variational segmentation framework as a decoupled approach, we are able to better preserve the connectivity of vascular networks than standard regularization terms. Our approach can be applied to unseen data and performs well, unlike deep learning segmentation approaches that have to be trained on each new dataset. Future work will extend our reconnecting term in 3D for the segmentation of various 3D vascular networks, where annotations are far more complicated to obtain. We will also modify our reconnecting network to learn a maximally monotone operator to ensure the convergence of our algorithm [16].

\section{REFERENCES}

[1] S. Moccia et al. "Blood vessel segmentation algorithms - review of methods, datasets and evaluation metrics". In: Comp. methods and programs in biomed. 158 (2018), pp. 71-91.

[2] O. Merveille et al. " $\mathrm{nD}$ variational restoration of curvilinear structures with prior-based directional regularization". In: Trans. on Image Processing (2019), pp. 3848 3859.

[3] A. Wu et al. "Deep vessel tracking: A generalized probabilistic approach via deep learning”. In: International Symposium on Biomedical Imaging. IEEE. 2016, pp. 1363-1367.
[4] M. Haft-Javaherian et al. "A topological encoding convolutional neural network for segmentation of 3D multiphoton images of brain vasculature using persistent homology". In: Computer Vision and Pattern Recognition Workshops. 2020, pp. 990-991.

[5] G. Tetteh et al. "Deepvesselnet: Vessel segmentation, centerline prediction, and bifurcation detection in 3-d angiographic volumes". In: Frontiers in Neuroscience 14 (2020).

[6] D. Gilton, G. Ongie, and R. Willett. "Neumann networks for linear inverse problems in imaging". In: Trans. on Comp. Imaging 6 (2019), pp. 328-343.

[7] F. Heide et al. "Flexisp: A flexible camera image processing framework". In: ACM Transactions on Graphics 33.6 (2014), pp. 1-13.

[8] A. Kofler et al. "Neural networks-based regularization for large-scale medical image reconstruction". In: Physics in Medicine \& Biology 65.13 (2020), p. 135003.

[9] T. Meinhardt et al. "Learning proximal operators: Using denoising networks for regularizing inverse imaging problems". In: International Conference on Computer Vision. 2017, pp. 1781-1790.

[10] T.F. Chan, S. Esedoglu, and M. Nikolova. "Algorithms for finding global minimizers of image segmentation and denoising models". In: SIAM journal on applied mathematics 66.5 (2006), pp. 1632-1648.

[11] E. Kerfoot et al. "Left-ventricle quantification using residual U-Net". In: International Workshop on Statistical Atlases and Computational Models of the Heart. 2018, pp. 371-380.

[12] A.D. Hoover, V. Kouznetsova, and M. Goldbaum. "Locating blood vessels in retinal images by piecewise threshold probing of a matched filter response". In: Trans. on Medical imaging 19.3 (2000), pp. 203-210.

[13] N. Komodakis and J.C. Pesquet. "Playing with duality: An overview of recent primal-dual approaches for solving large-scale optimization problems". In: Signal Processing Magazine 32.6 (2015), pp. 31-54.

[14] J. Staal et al. "Ridge-based vessel segmentation in color images of the retina". In: Trans. on Medical Imaging 23.4 (2004), pp. 501-509.

[15] M.E.G. Arias, I.P. Santos D.M. Borrero, and M..J.V. Vázquez. "A new deep learning method for blood vessel segmentation in retinal images based on convolutional kernels and modified U-Net model". In: Comp. Methods and Programs in Biomed. (2021), p. 106081.

[16] J.C. Pesquet et al. Learning Maximally Monotone Operators for Image Recovery. 2021. arXiv: 2012.13247. 